\title{
Cooper-pair propagation and superconducting correlations in graphene
}

\author{
J. González ${ }^{1}$ and E. Perfetto ${ }^{2}$ \\ ${ }^{1}$ Instituto de Estructura de la Materia, Consejo Superior de Investigaciones Científicas, Serrano 123, 28006 Madrid, Spain \\ ${ }^{2}$ Consorzio Nazionale Interuniversitario per le Scienze Fisiche della Materia, Università di Roma Tor Vergata, \\ Via della Ricerca Scientifica 1, 00133 Roma, Italy
}

(Received 25 May 2007; revised manuscript received 3 August 2007; published 3 October 2007)

\begin{abstract}
We investigate the Cooper-pair propagation and the Josephson effect in graphene under conditions in which the distance $L$ between superconducting electrodes is much larger than the width $W$ of the contacts. In the case of undoped graphene, we show that supercurrents may exist with a spatial decay proportional to $W^{2} / L^{3}$, reminiscent of the behavior of the critical current in disordered normal metals. We observe that there is in general a crossover temperature $T^{*} \sim v_{F} / k_{B} L$ that marks the onset of the strong decay of the supercurrent and that corresponds to the scale above which the Cooper pairs are disrupted by thermal effects during their propagation. We also show that the spatial decay of the critical current changes upon doping into a $1 / L^{2}$ behavior, opening the possibility to observe a supercurrent over length scales above $1 \mu \mathrm{m}$ at suitable doping levels.
\end{abstract}

DOI: 10.1103/PhysRevB.76.155404 PACS number(s): 81.05.Uw, 74.45.+c, 74.78.Na, 73.23. $-\mathrm{b}$

The recent interest in the physics of graphene has arisen from the observation of a number of novel electronic properties, ${ }^{1,2}$ which are the consequence of the relativisticlike character of the electron quasiparticles. This is certainly the case of the anomalous quantization of the Hall conductivity, ${ }^{3-5}$ as well as of the existence of a finite lower bound in the conductivity at the charge neutrality point. ${ }^{4,6-8}$ The graphene system is unique in that the low-energy excitations have conical dispersion around discrete Fermi points, being therefore governed by a Dirac equation for massless chiral particles. The appearance of an additional pseudospin quantum number intrinsic to the Dirac spectrum has led to propose other unconventional effects, such as the selective transmission of electrons through $n-p$ junctions ${ }^{9}$ or a peculiar form of Andreev reflection at the metal-superconductor interface in graphene. ${ }^{10}$

Recently, the proximity effect has been investigated in graphene in two different experiments. ${ }^{11,12}$ In both of them, a two-dimensional (2D) carbon layer has been contacted with superconducting electrodes, though the size and aspect ratio of the respective samples have been quite different in the two cases. Thus, while the separation of the electrodes in the experiment of Ref. 11 appears to be of the order of $\sim 0.5 \mu \mathrm{m}$, the distance between them in Ref. 12 seems to have a minimum value of about $2.5 \mu \mathrm{m}$. This may explain why a supercurrent has been measured between the electrodes in the first case, while the most relevant observation in the second experiment has been an abrupt drop in the resistance, below the critical temperature of the superconducting electrodes.

This crucial dependence on the distance between the electrodes may be, however, somewhat surprising, taking into account that supercurrents have been measured in carbon nanotubes placed between superconducting contacts, with nanotube lengths as large as $\approx 1.7 \mu \mathrm{m} .{ }^{13}$ It becomes then pertinent to analyze the proximity effect in graphene, especially for large separation between the superconducting electrodes, in order to find out the features related to the $2 \mathrm{D}$ character of the material.

The Josephson effect has been studied before in graphene strips with length $L$ small relative to their width and the superconducting coherence length. ${ }^{14}$ Here, we will face the opposite situation in which the relevant signatures are dictated by the propagation of the Cooper pairs in graphene. In this sense, we will adopt an approach complementary to that of the usual description in terms of Andreev reflection at the metal-superconductor interface. The analysis of the Cooperpair propagation will show to be convenient for the discussion of the many-body effects on the supercurrents over long distances, as the diagrammatics for the propagation of the Cooper pairs allow easily the introduction of interactions and thermal effects. Our conclusions will coincide with one aspect stressed in Ref. 14 regarding the fact that the longdistance decay of the supercurrents in a clean undoped graphene layer turns out to be the same as in a disordered normal metal. ${ }^{15}$ On the other hand, the stronger $1 / L^{3}$ decay that we find, in comparison with the case of carbon nanotubes and ribbons, will appear as a consequence of dealing with the propagation of the Cooper pairs over a large 2D layer, instead of being constrained within a reduced transverse dimension.

We investigate then the Josephson effect in a $2 \mathrm{D}$ graphene layer, under conditions in which the distance $L$ between the superconducting electrodes producing the Cooper pairs is much larger than the width $W$ of the contacts. More specifically, we will consider that the Hamiltonian for graphene with superconducting contacts along the coordinates $x_{1}=0$ and $x_{2}=L$ is given by

$$
\begin{aligned}
H= & v_{F} \int d^{2} r \Psi_{\sigma}^{(a) \dagger}(\mathbf{r}) \boldsymbol{\sigma}^{(a)} \cdot \partial \Psi_{\sigma}^{(a)}(\mathbf{r}) \\
& +\sum_{j=1,2} t \int_{0}^{W} d y \Psi_{\sigma}^{(a) \dagger}\left(x_{j}, y\right) \Psi_{S j, \sigma}\left(x_{j}, y\right)+\text { H.c. }
\end{aligned}
$$

(we work throughout the paper in units such that $\hbar=1$ ). In the above expression, $\boldsymbol{\sigma}^{(a)}$ are different sets of Pauli matrices for $a=1,2,{ }^{3}$ and a sum is implicit over the index $a$ accounting for the two different valleys and corresponding Dirac 
spinors $\Psi^{(a)}$ at opposite corners $K,-K$ in the graphene Brillouin zone. The spinor indices are omitted for simplicity, and a sum is also implicit over the spin index $\sigma$. Thus, we are going to assume that the electron fields $\Psi_{S 1}$ and $\Psi_{S 2}$ in the superconducting electrodes couple with the same amplitude to the two Dirac points and the two sublattices of the graphene layer. ${ }^{16}$ In this sense, we are considering a kind of ideal contact between graphene and the superconducting electrodes, which is realized, for instance, when the latter are on top of the 2D layer preserving the graphene lattice structure. In the continuum approximation implicit in the model [Eq. (1)], the interface between graphene and the superconducting electrodes takes place along a segment of width $W$ in each case, but we will consider otherwise that the sample of graphene is $2 \mathrm{D}$, with dimensions much larger than the width of the contacts in both the transverse and the longitudinal direction.

For our purposes, it will be enough to describe the superconductors in terms of the order parameter $\Delta$ and the normal density of states $\rho$. We are going to deal, in particular, with the case in which the time of propagation of the Cooper pairs between the contacts is much larger than $1 /|\Delta|$. This implies, equivalently, that the distance $L$ has to be much larger than the superconducting coherence length $\xi$. Then, a supercurrent may arise mainly from processes in which the Cooper pairs tunnel from one of the superconductors to the 2D layer, propagating to the other superconducting contact. Under the assumption of a relative large $|\Delta|$, we can make the approximation

$$
\left\langle\Psi_{S j, \sigma}\left(x_{j}, y ;-i \tau_{1}\right) \Psi_{S j,-\sigma}\left(x_{j}, y ;-i \tau_{2}\right)\right\rangle \approx e^{i \chi_{j}} \rho \delta\left(\tau_{1}-\tau_{2}\right),
$$

where the statistical averages, at temperature $T$, are taken over ordered products with respect to imaginary time $\tau$. The Josephson current $I_{s}$ can be computed as the derivative of the free energy with respect to the difference $\chi$ between the respective phases $\chi_{1}$ and $\chi_{2}$ of the order parameters in the two superconductors:

$$
I_{s}=2 e \frac{\partial}{\partial \chi} k_{B} T \log \left(\operatorname{Tr} e^{-H / k_{B} T}\right) .
$$

By expanding the free energy in the iteration of tunneling events from the second term of the Hamiltonian in Eq. (1), we see that the contributions to the Josephson current must arise from processes connecting the condensates of the two superconducting electrodes. The lowest nonvanishing order in the current is given by

$$
\begin{aligned}
I_{s} \approx & 2 e \frac{\partial}{\partial \chi} k_{B} T t^{4} \prod_{i=1}^{4} \int_{0}^{1 / k_{B} T} d \tau_{i} \int_{0}^{W} d y_{i} \\
& \times\left\langle\Psi_{S 1, \uparrow}\left(0, y_{1} ;-i \tau_{1}\right) \Psi_{S 1, \downarrow}\left(0, y_{2} ;-i \tau_{2}\right)\right\rangle \\
& \times\left\langle\Psi_{\uparrow}^{(a) \dagger}\left(0, y_{1} ;-i \tau_{1}\right) \Psi_{\downarrow}^{(-a) \dagger}\left(0, y_{2} ;-i \tau_{2}\right)\right. \\
& \left.\times \Psi_{\uparrow}^{(b)}\left(L, y_{3} ;-i \tau_{3}\right) \Psi_{\downarrow}^{(-b)}\left(L, y_{4} ;-i \tau_{4}\right)\right\rangle \\
& \times\left\langle\Psi_{S 2, \uparrow}^{\dagger}\left(L, y_{3} ;-i \tau_{3}\right) \Psi_{S 2, \downarrow}^{\dagger}\left(L, y_{4} ;-i \tau_{4}\right)\right\rangle .
\end{aligned}
$$

We will now take into account the assumption that $L \gg W$, averaging over the separation of the electrons in the pair tunneling along the superconducting contact. Making use of approximation (2) and of the translational invariance in the variable $\tau$, we obtain the following expression of the maximum supercurrent $I_{c}$ (critical current):

$$
\begin{aligned}
I_{c}(T) \approx & 2 e \rho^{2} t^{4} W^{2} \int_{0}^{W} d y_{1} \int_{0}^{W} d y_{2} \int_{0}^{1 / k_{B} T} d \tau \\
& \times\left\langle\Psi_{\uparrow}^{(a) \dagger}\left(0, y_{1} ; 0\right) \Psi_{\downarrow}^{(-a) \dagger}\left(0, y_{1} ; 0\right)\right. \\
& \left.\times \Psi_{\uparrow}^{(b)}\left(L, y_{2} ;-i \tau\right) \Psi_{\downarrow}^{(-b)}\left(L, y_{2} ;-i \tau\right)\right\rangle .
\end{aligned}
$$

The expression of the Cooper pairs that appear in Eq. (5) is a short notation for objects that are actually made of the tensor product of states of the electrons in the pair. The rule to build the state of the pair has to be consistent with the form of the tunneling Hamiltonian in Eq. (1), which implies, in particular, the choice of equal amplitudes for the two sublattices of the graphene layer. If we denote the operators corresponding to electron states with momentum $\mathbf{k}$ projected on the sublattice $A(B)$ by $\Psi_{A, \sigma}^{(a)}(\mathbf{k})\left[\Psi_{B, \sigma}^{(a)}(\mathbf{k})\right]$, a suitable expression of the Cooper pair is given by

$$
\Psi_{A, \uparrow}^{(K)}(\mathbf{k}) \Psi_{A, \downarrow}^{(-K)}(-\mathbf{k})+\Psi_{B, \uparrow}^{(K)}(\mathbf{k}) \Psi_{B, \downarrow}^{(-K)}(-\mathbf{k}) .
$$

This state can be cast in terms of the operators corresponding to electron states with well-defined energy in the conduction band, $\Psi_{+, \sigma}^{(a)}(\mathbf{k})$, and in the valence band, $\Psi_{-, \sigma}^{(a)}(\mathbf{k})$, given by

$$
\begin{gathered}
\Psi_{+, \sigma}^{(K)}(\mathbf{k})=\left(\Psi_{A, \sigma}^{(K)}(\mathbf{k})+e^{i \phi} \Psi_{B, \sigma}^{(K)}(\mathbf{k})\right) / \sqrt{2}, \\
\Psi_{-, \sigma}^{(K)}(\mathbf{k})=\left(\Psi_{A, \sigma}^{(K)}(\mathbf{k})-e^{i \phi} \Psi_{B, \sigma}^{(K)}(\mathbf{k})\right) / \sqrt{2}, \\
\Psi_{+, \sigma}^{(-K)}(-\mathbf{k})=\left(\Psi_{A, \sigma}^{(-K)}(-\mathbf{k})+e^{-i \phi} \Psi_{B, \sigma}^{(-K)}(-\mathbf{k})\right) / \sqrt{2}, \\
\Psi_{-, \sigma}^{(-K)}(-\mathbf{k})=\left(\Psi_{A, \sigma}^{(-K)}(-\mathbf{k})-e^{-i \phi} \Psi_{B, \sigma}^{(-K)}(-\mathbf{k})\right) / \sqrt{2},
\end{gathered}
$$

where $\phi=\arctan \left(k_{y} / k_{x}\right)$. It is easily seen that

$$
\begin{aligned}
& \Psi_{A, \uparrow}^{(K)}(\mathbf{k}) \Psi_{A, \downarrow}^{(-K)}(-\mathbf{k})+\Psi_{B, \uparrow}^{(K)}(\mathbf{k}) \Psi_{B, \downarrow}^{(-K)}(-\mathbf{k}) \\
& \quad=\Psi_{+, \uparrow}^{(K)}(\mathbf{k}) \Psi_{+, \downarrow}^{(-K)}(-\mathbf{k})+\Psi_{-, \uparrow}^{(K)}(\mathbf{k}) \Psi_{-, \downarrow}^{(-K)}(-\mathbf{k}) .
\end{aligned}
$$

It can be checked that the choice [Eq. (11)] for the state of the Cooper pairs leads to results that preserve the relativisticlike invariance of the theory, which is consistent with our assumption of ideal contacts to the superconducting electrodes maintaining the graphene lattice structure.

The determination of the critical current is therefore reduced to the evaluation of the propagator of the Cooper pairs in the state given by Eq. (11). The Fourier transform of the propagator at $T \neq 0$, which we will denote by $D(\mathbf{k}, i \bar{\omega})$, can be obtained from the standard diagrammatics for Dirac fermions. We stress that, for the sake of preserving the relativisticlike invariance, it is convenient to regularize the diagrams at high energies by using a method that maintains the space-time symmetry of the theory, like the analytic continuation in the number of dimensions. ${ }^{17}$ In particular, the Cooper-pair propagator can be obtained in the noninteracting theory from the convolution of two Dirac propagators, and at $T=0$, it turns out to be 

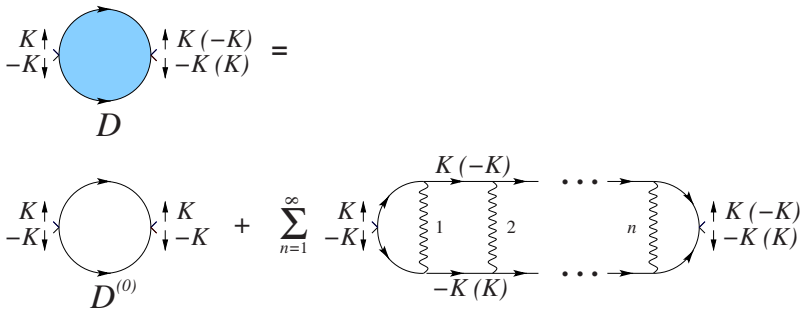

FIG. 1. (Color online) Diagrammatic equation for the Cooperpair propagator $D(\mathbf{k}, \omega)$, obtained as a sum of the iteration of scattering processes between the electrons in the pair.

$$
\left.D^{(0)}(\mathbf{k}, \omega)\right|_{T=0}=-\frac{1}{8 v_{F}^{2}} \sqrt{v_{F}^{2} \mathbf{k}^{2}-\omega^{2}} .
$$

The temperature dependence of the propagator can be obtained using the Matsubara formalism. In the static limit, we get

$$
\begin{aligned}
D^{(0)}(\mathbf{k}, 0)= & -\frac{1}{2 \pi v_{F}}|\mathbf{k}| \int_{0}^{1} d x \sqrt{x(1-x)} \tanh \left(\frac{v_{F}|\mathbf{k}| \sqrt{x(1-x)}}{2 k_{B} T}\right) \\
& -\frac{1}{\pi v_{F}^{2}} k_{B} T \int_{0}^{1} d x \ln \left[2 \cosh \left(\frac{\left.\left.v_{F}|\mathbf{k}| \sqrt{x(1-x)}\right)\right] .}{2 k_{B} T}\right)\right]
\end{aligned}
$$

The knowledge of the Cooper-pair propagator at zero frequency is enough to compute the critical current in Eq. (5). Interaction effects can be incorporated by summing up the different perturbative orders obtained by iteration of the scattering of the particles in the pair, as illustrated in Fig. 1. If we take an average of the potential $V$ between the particles, we have that the Cooper-pair propagator can be represented in the $T$-matrix approximation by

$$
D(\mathbf{k}, \omega) \approx \frac{D^{(0)}(\mathbf{k}, \omega)}{1+V D^{(0)}(\mathbf{k}, \omega)} .
$$

We remark anyhow that the interaction effects cannot significantly affect the propagation of the Cooper pairs at low temperatures. When there is exchange of the valleys in the scattering of the electrons in the pair, the Coulomb potential $V_{C}$ becomes suppressed by a large momentum transfer $2 k_{F}$, down to $V_{C} \sim e^{2} / 2 k_{F}$. At the small values of $|\mathbf{k}|$ relevant for the long-distance regime, the denominator in Eq. (14) remains then very close to 1 . In the opposite case of no valley exchange, we observe that at low momentum transfer, $V_{C} D^{(0)}$ has a relative strength of the order of $\sim e^{2} / v_{F}$. At low energies, this effective coupling is strongly renormalized, down to a value which may be about 1 order of magnitude below the nominal coupling. ${ }^{18}$ We have checked that, in practice, this makes the difference between computing the critical current [Eq. (5)] from either $D(\mathbf{k}, 0)$ or $D^{(0)}(\mathbf{k}, 0)$ very small at the relevant temperatures in the experiments (of the order of $\sim 1 \mathrm{~K}$ ).

It becomes convenient to factor out from Eq. (5) the relative tunnel conductances of the interfaces, which are each given by the constant quantity $\rho t^{2} W / v_{F} \cdot{ }^{19} \mathrm{We}$ will deal then

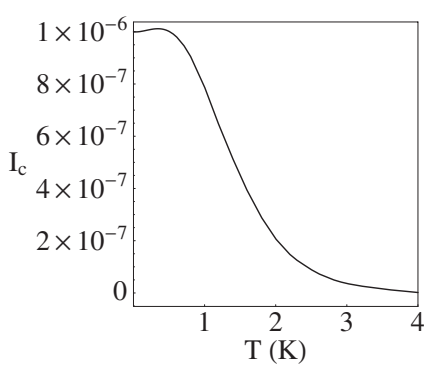

FIG. 2. Plot of the critical current $I_{c}^{(2 \mathrm{D})}(T)$ (in units of $\left.10^{-2} e v_{F} k_{c} \approx 1.2 \mu \mathrm{A}\right)$ for $L=5 \times 10^{3} / k_{c}(\approx 2.5 \mu \mathrm{m})$ and $W=10^{2} / k_{c}$ $(\approx 50 \mathrm{~nm})$.

with the intrinsic 2D dependence of the critical current, which becomes, in terms of the Fourier transform of the Cooper-pair propagator,

$$
I_{c}^{(2 \mathrm{D})}(T) \approx 2 e W^{2} v_{F}^{2} \int_{0}^{\infty} \frac{d k}{2 \pi}|\mathbf{k}| J_{0}(|\mathbf{k}| L) D(\mathbf{k}, 0) e^{-|\mathbf{k}| / k_{c}},
$$

where $k_{c}$ is a short-distance cutoff that we take in the order of $\sim 1 \mathrm{~nm}^{-1}$. This corresponds to $v_{F} k_{c} \sim 1 \mathrm{eV}$, which is the energy scale below which the Dirac theory applies to graphene. We stress that this energy is much larger than the thermal energy at the temperatures relevant for the experiments (of the order of $\sim 1 \mathrm{~K}$ ). Moreover, the scale $v_{F} k_{c}$ is also much larger than the low-energy scale $v_{F} / L$ at which we are going to compute the propagation of the Cooper pairs. This explains that, as we have checked explicitly, the results presented in what follows for the critical currents at $T \sim 1 \mathrm{~K}$ and distances well above the nanometer scale are very little sensitive to variations in $k_{c}$, as long as $v_{F} k_{c}$ remains orders of magnitude larger than any other energy scale in the model.

The critical current computed from Eq. (15) displays a number of peculiar features. From the scaling of $D^{(0)}(\mathbf{k}, 0)$ in the zero-temperature limit, it is easily seen that $I_{c}^{(2 \mathrm{D})}(0)$ must decay as $\sim v_{F} W^{2} / L^{3}$ at large separation between the contacts. Another important property is that there is always a crossover temperature $T^{*} \sim v_{F} / k_{B} L$, which marks the onset of a power-law decay of the supercurrent at high temperatures. This is shown in Fig. 2, which represents the behavior of the critical current as a function of $T$ for $L=5 \times 10^{3} / k_{c}$ and $W$ $=10^{2} / k_{c}$, taking $k_{c}=2 \mathrm{~nm}^{-1}$. The dependence of $I_{c}^{(2 \mathrm{D})}(T)$ is qualitatively similar to what is found in carbon nanotubes with long separation between superconducting contacts. ${ }^{20,21}$ The crossover temperature corresponds to the scale at which the Cooper pairs fail to propagate efficiently between the electrodes, as they become disrupted along the way by thermal effects.

The different behavior of the supercurrents in graphene with respect to that in the carbon nanotubes comes from the scaling with length $L$ at low temperatures. This is given by a $1 / L^{3}$ power-law behavior in graphene, instead of the $1 / L$ scaling of a noninteracting one-dimensional (1D) system. ${ }^{20}$ We may consider, for instance, the sample described in Ref. 12, with an approximate separation between the electrodes 


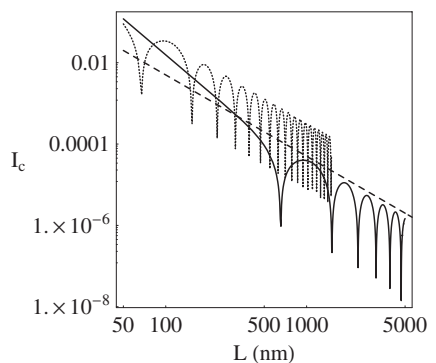

FIG. 3. Plot of the zero-temperature critical current $I_{c}^{(2 \mathrm{D})}(0)$ (in units of $10^{-2} e v_{F} k_{c} \approx 1.2 \mu \mathrm{A}$ ) as a function of the distance $L$ (keeping $W=10^{2} / k_{c} \approx 50 \mathrm{~nm}$ ) for chemical potentials $\mu=1 \mathrm{meV}$ (full line) and $\mu=10 \mathrm{meV}$ (dotted line). The dashed line corresponds to a $1 / L^{2}$ decay and it is drawn here as a reference to that power-law behavior.

$L \approx 2.5 \mu \mathrm{m}$. The plot in Fig. 2 gives the theoretical values of the critical current, in units of $10^{-2} e v_{F} k_{c} \approx 1.2 \mu \mathrm{A}$. We observe that, in the low-temperature regime to the left of the crossover, the expected critical currents should have a magnitude $I_{c}^{(2 \mathrm{D})} \sim 10^{-3} \mathrm{nA}$. Such a small scale may explain the difficulty in establishing a supercurrent in graphene when there is a separation of the order of microns between the superconducting electrodes.

On the other hand, the crossover shown in Fig. 2 has a remarkable correspondence with the abrupt drop in the resistance reported in Ref. 12. This feature has been observed at a temperature of about $1 \mathrm{~K}$, and it does not seem to bear a direct relation to the critical temperature $T_{c}(\approx 4 \mathrm{~K})$ of the superconducting electrodes. We observe that the crossover displayed in Fig. 2 corresponds to a temperature of about
$1.5 \mathrm{~K}$, in fair agreement with the position of the drop measured experimentally in the resistance. It is therefore quite likely that this feature may be an indirect signature of the temperature up to which the Cooper pairs are able to propagate without disruption, along the $2.5-\mu \mathrm{m}$-long path of the sample reported in Ref. 12.

The fast decay of the supercurrent computed from Eq. (15) can be traced back to the vanishing density of states of graphene at the charge neutrality point. Actually, the form of the propagator in Eq. (12) is a direct consequence of the conical dispersion around the Fermi points of graphene. One can therefore expect important changes in the Cooper-pair propagation upon doping the electron system. This can be investigated formally by introducing a finite chemical potential $\mu$, with the aim of shifting the Fermi level away from the charge neutrality point. At $\mu>0$, the Dirac propagator can be written as ${ }^{22}$

$$
\begin{aligned}
G^{(0)}(\mathbf{k}, \omega)= & \left(\omega+v_{F} \boldsymbol{\sigma} \cdot \mathbf{k}\right)\left[\frac{-1}{-\omega^{2}+v_{F}^{2} \mathbf{k}^{2}-i \epsilon}\right. \\
& \left.+i \pi \frac{\delta\left(\omega-v_{F}|\mathbf{k}|\right)}{v_{F}|\mathbf{k}|} \theta\left(\mu-v_{F}|\mathbf{k}|\right)\right],
\end{aligned}
$$

which is a convenient alternative form of expressing the electron propagator

$$
G^{(0)}(\mathbf{k}, \omega)=\frac{\theta(\varepsilon(\mathbf{k})-\mu)}{\omega-\varepsilon(\mathbf{k})+i \epsilon}+\frac{\theta(\mu-\varepsilon(\mathbf{k}))}{\omega-\varepsilon(\mathbf{k})-i \epsilon}
$$

for a dispersion with two branches $\varepsilon(\mathbf{k})= \pm v_{F}|\mathbf{k}|$. The Cooper-pair propagator can now be computed from the convolution of two Dirac propagators like Eq. (16). The result at $\omega=0$ is

$$
D^{(0)}(\mathbf{k}, 0)= \begin{cases}-\frac{1}{2 \pi v_{F}^{2}} \mu & \text { if }|\mathbf{k}|<2 \mu \\ -\frac{1}{8 v_{F}}|\mathbf{k}|+\frac{1}{4 \pi v_{F}}|\mathbf{k}| \arcsin \left(\frac{2 \mu}{|\mathbf{k}|}\right)-\frac{1}{2 \pi v_{F}^{2}} \mu & \text { if }|\mathbf{k}|>2 \mu .\end{cases}
$$

The slight change produced by the chemical potential in the infrared behavior of $D^{(0)}(\mathbf{k}, 0)$ is enough to modify the long-distance decay of the supercurrent. We have represented in Fig. 3 the result of evaluating $I_{c}^{(2 \mathrm{D})}(0)$ from Eq. (15) with the Cooper-pair propagator in Eq. (18). It can be seen that, at $L \sim v_{F} / \mu$, the power-law decay of the critical current changes from $1 / L^{3}$ to $1 / L^{2}$. The supercurrents cannot be enhanced anyhow up to the magnitudes that they reach in a $1 \mathrm{D}$ electron system, where the decay is given by a $1 / L$ dependence in the noninteracting theory. However, it may be worthwhile to explore experimentally the consequences of doping the graphene layer, up to levels where it can be affordable to measure supercurrents of the order of $\sim 1 \mathrm{nA}$ for suitably small values of $L$, according to the plot shown in Fig. 3.

We also remark that doping the graphene layer may be the way to obtain experimental signatures of dynamical superconducting correlations. The pertinent approach to address this question is to deal again with the theory at nonvanishing chemical potential, starting from the Dirac propagator in Eq. (16). Computing now the Cooper-pair propagator at real frequency $\omega$, the possible superconducting instabilities have to reflect as singularities in $D(0, \omega)$, as it happens with the formation of bound electron pairs in the BCS theory. ${ }^{23}$

The evaluation of the Cooper-pair propagator at chemical potential $\mu>0$ gives the result 


$$
D^{(0)}(0, \omega)=-\frac{1}{4 \pi v_{F}^{2}} \omega \log \left|\frac{2 \mu-\omega}{\omega}\right|-\frac{1}{2 \pi v_{F}^{2}} \mu-i \frac{1}{8 v_{F}^{2}} \omega .
$$

We may sum up again multiple scattering processes of the Cooper pairs in the framework of the $T$-matrix approximation. We observe then that $D(0, \omega)$ has a pole under conditions where the dominant interaction is attractive, such that $V<0$. The denominator $1+V D^{(0)}(0, \omega)$ in Eq. (14) vanishes at values of the frequency $\omega$ given by the equation

$$
|2 \mu-\omega|=\omega \exp \left(\frac{4 \pi v_{F}^{2}-2 \mu V}{\omega V}\right) .
$$

There is always a solution of Eq. (20) for $\omega$ below and close to $2 \mu$ when $V<0$. Such a frequency marks the formation of bound electron pairs that may take place for arbitrarily small values of $\mu$, despite the fact that the density of states of graphene vanishes at $\omega=0 .{ }^{24}$ The main difference with a conventional Fermi liquid is that the energy scale in front of the exponential in Eq. (20) may be rather small, of the order of $\sim 2 \mu$.

In conclusion, we have studied the Josephson effect in a 2D graphene layer when the distance between the superconducting contacts is much larger than their width. We have seen that, in the case of undoped graphene, the supercurrents have a fast spatial decay, proportional to $W^{2} / L^{3}$. This strong dependence on $L$ reminds of the behavior of the critical cur- rent in long diffusive junctions. ${ }^{25}$ The similarity between the behavior of ballistic graphene and that of a disordered normal metal has also been noted in the study of transport in short graphene strips. ${ }^{7,14}$

On the other hand, it is unlikely that the experimental measures may be strongly affected, in general, by disorder in a graphene layer, since backscattering arises only from small-range scatterers with a size not larger than the lattice constant. ${ }^{26}$ We have actually seen that the results reported in Ref. 12 are consistent with a crossover to the strong decay of the critical current at a temperature $T^{*} \sim v_{F} / k_{B} L$, and not at the much smaller scale given in a diffusive junction in terms of the diffusion constant $\mathcal{D}$ by $T^{*} \sim \mathcal{D} / k_{B} L^{2}$.

We have seen that shifting the Fermi level away from the charge neutrality point changes the dependence of the critical current into a $1 / L^{2}$ behavior, opening the possibility to observe a supercurrent over length scales above $1 \mu \mathrm{m}$ at suitable doping levels. We have shown that this becomes feasible anyhow below the crossover temperature $T^{*}$ marking the onset of the power-law decay of the supercurrent and corresponding to the scale above which the Cooper pairs are increasingly disrupted by thermal effects during their propagation.

We thank H. Bouchiat and F. Guinea for useful comments on the paper. The financial support of the Ministerio de Educación y Ciencia (Spain) through Grant No. FIS2005-05478C02-02 is gratefully acknowledged. E.P. is also financially supported by CNISM (Italy).
${ }^{1}$ K. S. Novoselov, A. K. Geim, S. V. Morozov, D. Jiang, M. I. Katsnelson, I. V. Grigorieva, S. V. Dubonos, and A. A. Firsov, Nature (London) 438, 197 (2005).

${ }^{2}$ Y. Zhang, Y.-W. Tan, H. L. Stormer, and P. Kim, Nature (London) 438, 201 (2005).

${ }^{3}$ Y. Zheng and T. Ando, Phys. Rev. B 65, 245420 (2002).

${ }^{4}$ N. M. R. Peres, F. Guinea, and A. H. Castro Neto, Phys. Rev. B 73, 125411 (2006).

${ }^{5}$ V. P. Gusynin and S. G. Sharapov, Phys. Rev. Lett. 95, 146801 (2005).

${ }^{6}$ M. I. Katsnelson, Eur. Phys. J. B 51, 157 (2006).

${ }^{7}$ J. Tworzydlo, B. Trauzettel, M. Titov, A. Rycerz, and C. W. J. Beenakker, Phys. Rev. Lett. 96, 246802 (2006).

${ }^{8}$ K. Nomura and A. H. MacDonald, Phys. Rev. Lett. 98, 076602 (2007).

${ }^{9}$ V. V. Cheianov and V. I. Fal'ko, Phys. Rev. B 74, 041403(R) (2006).

${ }^{10}$ C. W. J. Beenakker, Phys. Rev. Lett. 97, 067007 (2006).

${ }^{11}$ H. B. Heersche, P. Jarillo-Herrero, J. B. Oostinga, L. M. K. Vandersypen, and A. F. Morpurgo, Nature (London) 446, 56 (2007).

${ }^{12}$ A. Shailos, W. Nativel, A. Kasumov, C. Collet, M. Ferrier, S. Guéron, R. Deblock, and H. Bouchiat, Europhys. Lett. 79, 57008 (2007).

${ }^{13}$ A. Yu. Kasumov, R. Deblock, M. Kociak, B. Reulet, H. Bouchiat, I. I. Khodos, Yu. B. Gorbatov, V. T. Volkov, C. Journet, and M. Burghard, Science 284, 1508 (1999).
${ }^{14}$ M. Titov and C. W. J. Beenakker, Phys. Rev. B 74, 041401(R) (2006).

${ }^{15}$ This aspect of transport in graphene has been noted in a different context in Ref. 7.

${ }^{16}$ The amplitudes of the electrons in the two sublattices of graphene are given by the upper and lower components of the spinors, in the notation used to write Eq. (1).

${ }^{17}$ J. González, F. Guinea, and M. A. H. Vozmediano, Nucl. Phys. B 424, 595 (1994).

${ }^{18}$ J. González, F. Guinea, and M. A. H. Vozmediano, Phys. Rev. B 59, R2474 (1999).

${ }^{19}$ It can be easily seen that the relative conductance $\rho t^{2} W / v_{F}$ is a dimensionless quantity, as the electron fields $\Psi^{(a)}$ in graphene have the dimension of the inverse of a length according to Eq. (1).

${ }^{20}$ J. González, Phys. Rev. Lett. 87, 136401 (2001).

${ }^{21}$ J. González, J. Phys.: Condens. Matter 15, S2473 (2003).

${ }^{22}$ See, for instance, S. A. Chin, Ann. Phys. (N.Y.) 108, 301 (1977).

${ }^{23}$ A. A. Abrikosov, L. P. Gorkov, and I. E. Dzyaloshinski, Methods of Quantum Field Theory in Statistical Physics (Dover, New York, 1975), Chap. 7.

${ }^{24}$ B. Uchoa and A. H. Castro Neto, Phys. Rev. Lett. 98, 146801 (2007).

${ }^{25}$ P. Dubos, H. Courtois, B. Pannetier, F. K. Wilhelm, A. D. Zaikin, and G. Schön, Phys. Rev. B 63, 064502 (2001).

${ }^{26}$ T. Ando, J. Phys. Soc. Jpn. 74, 777 (2005). 